\title{
A COMPLETE SENSOR-BASED SYSTEM TO NAVIGATE THROUGH A CLUTTERED ENVIRONMENT
}

\author{
A. Durand-Petiteville ${ }^{1}$, V. Cadenat ${ }^{2}$ and N. Ouadah ${ }^{3}$ \\ ${ }^{1}$ University of California, Davis, Department of Biological and Agricultural Engineering, One Shields Avenue, Davis, CA \\ 95616-5270, U.S.A \\ ${ }^{2}$ CNRS, LAAS, 7 avenue du colonel Roche, F-31400 Toulouse, France \\ Univ de Toulouse, UPS, LAAS, F-31400, Toulouse, France \\ ${ }^{3}$ Centre de Développement des Technologies Avancées \\ Cité du 20 Août 1956, BP 17 Baba Hassen, 16303 Algiers, Algeria \\ adurandp@ucdavis.edu,cadenat@laas.fr,ouadah@cdta.dz
}

Keywords: Sensor-based Navigation, Mobile Robot, Topological Map.

Abstract: This article deals with the autonomous navigation problem of a mobile robot in a cluttered environment. We propose to have a different perspective than the traditional way of splitting the problem into two categories: the map-based ones and the mapless ones. Here we divide navigation systems into six processes: perception, modeling, localization, planning, action and decision. Then we present how those processes are organized into an architecture to perform a navigation. It is shown that this framework embraces any navigation system proposed in the literature and how it allows to create new combination of processes. We then detail our solution to the problem which mainly consists in coupling sensor-based controllers with a topological map. Moreover we present the used tools that we have developed over the last years as well as the ones from the literature. Finally we present experimentation results of a long-range navigation based on the proposed approach where a robot drives through an environement despite of occlusions and possible collisions due to obstacles.

\section{INTRODUCTION}

In this paper, we consider the autonomous navigation problem of a mobile robot in a cluttered environment. It consists in reaching a goal through a given environment while dealing with unexpected events mainly due to obstacles (Choset et al., 2005). To do so, different sensors have been used and a wide variety of techniques have been proposed in the literature. Among them, the strategies based on vision have significantly drawn the interest of researchers (BoninFont et al., 2008). Here, we focus on particular visual navigation approaches consisting in coupling sensorbased controllers to a topological map. Indeed they seem to be appropriate to our problem for two reasons. First they allow to deal with unexpected events thanks to the reactive behavior of the sensor-based controllers. Second, it is possible to navigate safely over large distances using the map. The literature provides several approaches relying on a similar reasoning. Among them, we focus on the works based on the concept of visual route (Matsumoto et al., 1996). This latter is a topological map built by organizing im- ages taken during a pre-navigation step (Royer et al., 2007). It is then coupled with a controller allowing to make the robot move along this road. See for example the works by (Booij et al., 2007) (Courbon et al., 2009) for omni-directional vision systems and by (Krajník and Přeučil, 2008) (Courbon et al., 2009) for pinhole cameras. Note that in these works vision is used to build the topological map and to reconstruct the robot state value. This choice prevents from keeping the well known advantages of imagebased visual servoing in terms of robustness and precision (Chaumette and Hutchinson, 2006). Moreover, none of these approaches takes into account the two major problems of visual navigation: occlusions, i.e. the landmarks loss, and collisions with obstacles. A set of works (Cherubini et al., 2011) (Cherubini and Chaumette, 2013) have recently developed a visual navigation strategy allowing to avoid unexpected obstacles while tolerating partial occlusions.

We believe that these weaknesses come from the fact that the traditional framework proposed in the literature does not provide sufficient guidelines to build a navigation system. In this framework (Choset et al., 
2005) (Bonin-Font et al., 2008), the strategies are classically split into two categories: the map-based ones and the mapless (or reactive) ones. The first ones allow to realize large displacements while suffering from a lack of flexibility when dealing with unmapped obstacles and some sensitivity in the presence of errors. The second ones allow to handle the unexpected events much more efficiently than the previous ones but they are not adapted to perform long displacements. Thus, the traditional formalism does not cover the visual navigation strategies we are interested in, as they cumulate the advantages of both local and global approaches. Furthermore, as previously mentioned, it does not provide sufficient guidelines to help the strategy design. This is due to the fact that the framework categorizes navigation methods using only one criterion: the presence or not of a priori data about the environment, which is limited. For us, it appears to be worthwhile to analyze the problem using a different perspective. To do so, we propose to describe the navigation strategy using the six processes which are involved in it, namely: perception, modeling, planning, localization, action and decision. These processes are in turn organized into an architecture which indicates which ones are used and how they interact together. Thus, to design a navigation system, it is necessary to select a method allowing to instantiate each process. The system is then defined using the methods involved in each process.

Based on that navigation framework, we have worked over the last decade in order to create a complete navigation system allowing to visually navigate through a cluttered environment using sensor-based controllers. Indeed, we believe that those controllers, thanks to their nice reactive behavior, allow to efficiently guide a robot towards a goal while dealing with obstacles. To design such a system, we have developed methods to avoid obstacles based on laser range-finder data (Souères et al., 1998), to switch from one controller to another one depending on the needs (Souères and Cadenat, 2003), to reconstruct visual features in order to manage occlusions (Durand Petiteville et al., 2013). In this paper we detail the instantiation of each process and describe how they are organized in the chosen architecture. Finally, thanks to experimentations, we show that the proposed navigation system allows to deal efficiently with unexpected events such as visual signal losses or obstacles, contrary to most other similar techniques.

This paper is organized as follows. The next section is devoted to the navigation framework. Then, we present our approach in part 3, highlighting the choices we have made for the processes. We end by showing results validating the proposed approach.

\section{THE NAVIGATION FRAMEWORK}

Six processes are generally involved in a given navigation system: perception, modeling, planning, localization, action and decision. The level of autonomy and the performances of the system can be evaluated only after having considered each of those processes. This is the reason why we believe that it is worthwhile to analyze a navigation through these processes. In this section we exhibit different architectures describing the processes and their collaboration. We consider several scenarii and for each of them we describe the corresponding architecture. It will be shown that any navigation approach can match the proposed framework, demonstrating its generality. We propose to organize our presentation around the choice of the controller allowing to drive the robot towards the goal.

\section{1 "State feedback" based controller}

We consider a robot driven to its goal thanks to a state feedback controller as its action process. To compute the control inputs, the state value in the world frame has to be known at any time, which means that the localization process has to be instantiated with a metric localization. The robot capacity to geometrically localize itself is then a necessary condition to successfully perform the navigation. Let us note that the distance that the robot can cover is significantly limited by the localization precision. Indeed, a too large error on the state value will result in inconsistent control inputs.

We now deal with the collisions problem due to obstacles presence in the environment. In this case there are two solutions, either based on the action or the planning processes. The action one consists in controlling the robot using two controllers: a first one making the error between the current and the desired poses vanish, allowing to reach the goal, and a second one performing the obstacle avoidance using exteroceptive data. It is then necessary to instantiate the decision process with a supervision module able to select the adequate controller. This solution, which guarantees the non-collision with obstacles, does not allow to ensure the navigation success. Indeed, the obstacle avoidance is locally performed and does not take into account the goal. Some local minima might arise. The second solution consists in following a previously planned collision free path. To achieve this aim, we have to use the modeling process. If the model represents the whole scene, then the navigation simply consists in following the planned itinerary us- 
ing a state feedback controller. The decision process is no more required. If the environment is not completely modeled, it may be necessary to update the map when an obstacle appears on the robot path. After the update, a re-planning step is performed. In this case, the decision process which decides when updating and re-planning is mandatory. Finally, it should be noticed that the metric localization is required and limits the navigation range for each solution.

\section{2 "Output feedback" based controller}

We now consider a robot moving towards its goal using an output feedback controller as its action process. The initial pose is unknown whereas the desired one is defined by measures with respect to a landmark. The robot can converge toward the desired pose if the landmark can be perceived at any instant. It is now the sensor range which limits the navigation range. In this case no localization process is required.

We now focus on the collisions problem. A first solution consists in using a sole output feedback controller to reach the desired pose while avoiding obstacles. A second idea is to add another controller in the action process. It has to guarantee the non collision with obstacle. A decision process selecting the adequate controller is then required. For both solutions, local minima problems may occur. Moreover, the navigation range is still limited by the sensors range. A modeling process containing global informations must then be used to perform a long range navigation. The global information can be added using a metric map or a topological map. In the first case, the planning process can plan a path taking into account the features availability at each pose. The planned itinerary is then composed by several landmarks successively used to compute the control inputs. Moreover, for a static environment, joint limits, visibility and obstacles can also be considered in the planning step. Nevertheless, this approach requires environment, robot and sensors reliable models. In the second case, a topological map is used to provide the necessary global information. Here, the additional data associated to the graph nodes usually correspond to the desired features or landmarks. As previously, the planned itinerary is made of measures or landmarks set to reach. This approach is based on a partial environment representation. The model is then less sensitive to the environment modifications, but does not allow to take into account several constraints such as obstacles or joints limits during the planning step. A topological localization is needed.

As we have seen, a navigation system does not solely depend on the presence or not of a map to de- scribe the environment, and trying to establish a classification using this sole criterion appears to be limited. On the contrary, considering the six processes and organizing them into an architecture allows to describe any navigation system and much more provides useful guidelines to build an efficient solution. Indeed, it becomes possible to create any combination of processes and instantiations in order to obtain robust navigation systems. Following this idea, we now present our own solution to the navigation problem.

\section{OUR SENSOR-BASED NAVIGATION STRATEGY}

In this section, we present our sensor-based solution to make a robot navigate through a cluttered environment. We first introduce the considered robotic system, then the architecture, and finally we detail how each process has been instantiated.

\subsection{The robotic system}

In this paper we consider an iRobot B21R mobile platform, which is a differential robot (see figure 1(a)). Its standard equipment has been extended with a vision system composed of a digital Point Grey CCD camera, mounted on a Directed Perception Pan Tilt Unit (PTU). Only the pan degree of freedom will be used in our experiments. The camera is able to provide 640x480 RGB images. A short range SICK laser range finder has been fixed at the bottom of the mobile base. It is able to detect obstacles up to 10 meters with a 180 degrees field of view and its resolution is about 1.9 points per degree. Sensors acquisitions and actuators management are performed thanks to an open source tool developed in LAAS which uses a $\mathrm{C} / \mathrm{C}++$ interfacing scheme.

\subsection{The navigation architecture}

Our architecture is based on the previous analysis and uses the introduced framework. To instantiate the perception, action and modeling processes, we have chosen to use a camera and a laser range finder, sensorbased-controllers and a topological map. Several reasons motivate this choice. Firstly, as explained in the introduction, vision has been widely exploited for performing various tasks (manipulation, navigation, etc.) and for different kinds of robots (humanoids, autonomous ground vehicles, etc.) (Bonin-Font et al., 2008). It is then worthwhile to consider this sensor to guide our robot during the navigation. Furthermore we have decided to couple it with a laser range- 


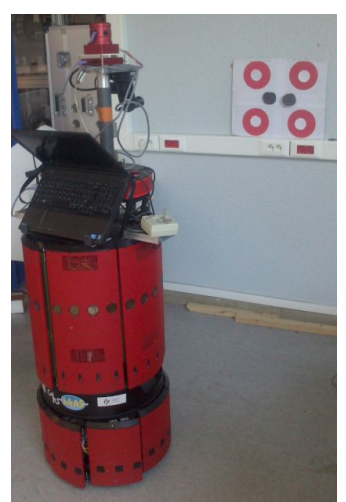

(a) The robot and the target.

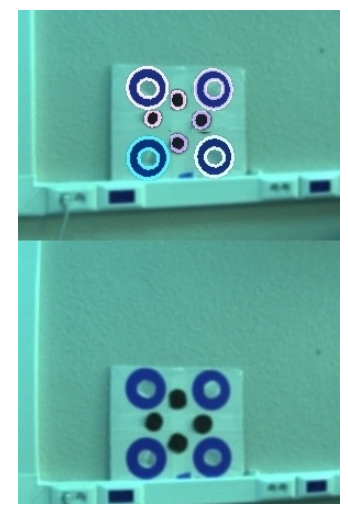

(b) The detection of the circles of interest and of the pattern.
Figure 1: The experimental set-up and the environment.

finder because such a device allows to efficiently detect obstacles and to provide accurate data to avoid them. Secondly, we have chosen to instantiate the action process using sensor-based controllers. Indeed, they are suitable to deal with unexpected events such as unmapped obstacles due to their reactivity aspect. Moreover, they do not require any metric localization, which allows to reduce the navigation failure risk due to a localization error. Finally, the environment has been represented by a topological map because this model presents several advantages with respect to its metric alter-ego, namely: (i) the metric localization is no more required, (ii) it provides sufficient data to perform a navigation task, without significantly increasing the problem complexity and (iii) it is less sensitive to scene modifications. Now we focus on the localization and planning processes. To instantiate them, we have selected methods matching the previous choices done for them. As we plan to use a topological map, we have chosen to instantiate the planning process by an algorithm from graph theory, and the localization process by a topological localization method. Finally, the decision process will be performed by supervision graphs which will define how each process runs with respect to the global strategy. Thanks to the new framework, our navigation architecture owns the advantages of both map-based and reactive strategies.

\subsection{The navigation processes}

\subsubsection{Perception}

The robot navigates using artificial landmarks which are fixed in the environment in such a way that a topological map can be built. These landmarks are made of a set of colored circles together with a particular
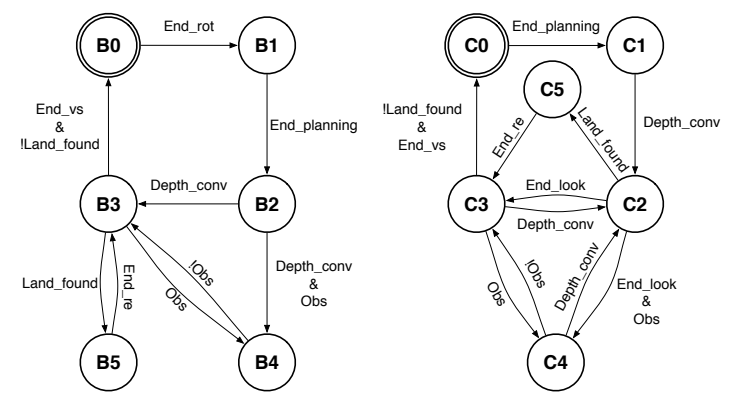

(a) The mobile base super- (b) The camera supervision vision process

process

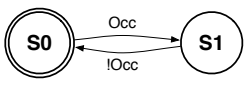

(c) The perception supervision process

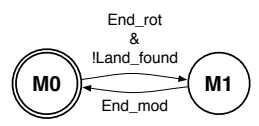

(e) The modeling supervision process

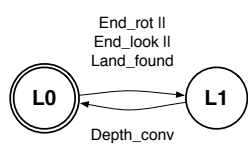

(d) The localization supervision process

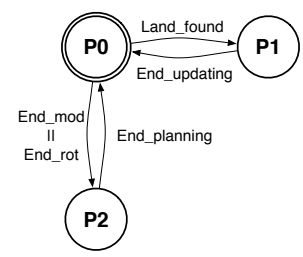

(f) The planning supervision process
Figure 2: The supervision graphs $(!:$ not $-\|:$ or $-\&$ : and $)$
B0 : Rotation
B1 : Nothing
B2 : Initialization
B3 : Visual servoing
B4 : Obstacle avoidance
B5 : Re-orientation
C0 : Nothing
C1 : Initialization
C2 : Looking for the next target
C4 : Obstacle avoidance
C3 : Visual servoing
S0 : Measured visual features
$\&$ laser rangefinder
S1 : Estimated visual features
\& laser rangefinder
L0 : Identify current landmark
L1 : Nothing
M0 : Nothing
M1 : Suppress a connection
P0 : Nothing
P2 : Plane a path
P1 : Update the current landmark

pattern in the center (figure 1(a)). The red and white circles are used to feed the control laws, while the pattern, made of several black circles in the middle of the target, allows to identify the target and localize the robot in the topological graph. The first objective of the image processing is to extract the center of the red and black circles. It relies on two criteria respectively based on color and on shape. The proposed image processing technique allows to detect $60 \times 60 \mathrm{~cm}$ tar- 
gets (Figure $1(\mathrm{~b}))^{1}$ up to 10 meters.

The robot is also equipped with a laser range finder allowing to detect the obstacles around the mobile base by providing a local scan of the environment. These data are then used to evaluate if an obstacle is close enough to be considered as dangerous. In such a case, they are also required to compute the control law allowing to avoid the considered obstacle.

During the navigation, several phenomenons can lead to the lack of visual features: camera temporary breakdown, image processing errors, or even occlusions of the landmarks by an obstacle. To success the mission, a method managing the loss of the visual signal is required. We have developed an algorithm (Durand Petiteville et al., 2013) which allows to reconstruct the point visual features and their depth in the general case of a 6 degrees of freedom calibrated camera. While visual data are available, the algorithm uses a number $n$ of images to estimate the current depth of each visual features. If the visual signal is lost, the last estimated depth is used as an initial value to predict the current one. It is then possible to estimate the current visual features. They are used to feed the control law allowing to perform the task. It then becomes possible to manage a total visual signal loss during the navigation.

\subsubsection{Modeling}

We now focus on the modeling process implementation. As previously mentioned, it is instantiated by a topological map, which consists of a directed graph. To build it, we have first chosen to associate to each node a landmark present in the scene. Thus, if there are $n_{l}$ landmarks, then the graph is composed of $n_{l}$ nodes. To connect the nodes, we detect if there exists a common visibility area, i.e. if two landmarks can be seen simultaneously from a given robot position. To achieve this aim, we associate to the node $N_{i}$, with $i \in\left[1, \ldots, n_{l}\right]$, several robot poses denoted by $S_{i-j}^{*}$, with $j \in\left[1, \ldots, n_{l}\right]$ and $i \neq j . S_{i-j}^{*}$ corresponds to the pose associated to the landmark $T_{i}$ that the robot should reach if it has then to navigate with respect to the landmark $T_{j}$. If $S_{i-j}^{*}$ is identical for all $T_{j}$, then it is denoted by $S_{i}^{*}$. An $\operatorname{arc} A\left(N_{i}, N_{j}\right)$ is then created if the landmark $T_{j}$, associated to the node $N_{j}$, can be seen from the pose $S_{i-j}^{*}$, associated to the node $N_{i}$, with $i \neq j$. Finally, we also associate to the node $N_{i}$ sensory data $D_{i}$ allowing to identify landmark $T_{i} . D_{i}$ is defined by the number of black circles in the middle of the target.

\footnotetext{
${ }^{1}$ The red circles diameter is $30 \mathrm{~cm}$ and the distance between two circles is about $40 \mathrm{~cm}$.
}

\subsubsection{Localization}

During the navigation the robot has to localize itself in the graph. We then need to use a topological localization method. The process consists in matching the data perceived by the sensors with the ones contained in the topological map (Segvic et al., 2009). Here, the process has to identify the landmarks in the field of view of the camera. They are made of black circles dedicated to localization. Those latter are identified thanks to image processing and the obtained result is matched to the database. Thus the robot knows which landmark is lying in the camera field of view and therefore where it is in the graph.

\subsubsection{Planning}

The planning process has to provide a path allowing to reach the goal from the initial robot configuration. To do so, the first step consists in finding the initial and final nodes. The first one is obtained during the initial localization step during which the robot makes a whole turn on itself to scan the environment. Each landmark that is seen is considered as a possible initial node. The final node, the goal to be reached, is given by the user. Then, for each initial node, we compute the shortest path using the Dijkstra algorithm. Finally, we keep the shortest path. The obtained path $T_{P}$ is therefore made of a sequence of $n_{P}$ landmarks $\left[T_{P 1}, \ldots, T_{P n_{P}}\right]$ to be reached successively.

\subsubsection{Action}

We have designed five output feedback controllers to successfully realize the mission. We have used the task function approach (Samson et al., 1991), where each considered task is modeled by a particular function, the so-called task function. This latter must be chosen so that it is zero when the mission is successfully performed. It then suffices to design a controller making the corresponding task function vanish. We propose to impose an exponential decrease to the task function as it is done in (Cadenat et al., 2012).

The first controller allows the robot to reach a pose with respect to a given landmark. This latter is described by a set of point-wise visual features which can be by the vision system. We have chosen to design an Image Based Visual Servoing (IBVS) (Chaumette and Hutchinson, 2006). This controller allows to make the current visual features converge towards a preselected reference value which corresponds to the one obtained at the desired pose.

The second controller is intended to guarantee non collision by allowing the vehicle to follow a security envelope around an obstacle. To achieve this aim, we 
have used the path following controller introduced in (Cadenat et al., 2012). It should be noticed that this controller allows to drive the mobile base only. Indeed, during the avoidance phase, the pan-platform is driven separately to keep the landmark of interest in the camera field of view. This is the reason why we have decoupled the motions of the mobile base and the pan-platform during this phase. We have used the controller proposed in (Durand Petiteville et al., 2011) which allows to maintain the target in the center of the image while compensating the mobile base motion.

The fourth controller is the reorientation one which intends to answer some problems due to the use of IBVS (Durand Petiteville et al., 2011). They may occur when switching from the current landmark to the next one. We have defined a controller allowing to suitably orientate the mobile base toward the next target when it has been detected (Durand Petiteville et al., 2011). When the camera is orientated towards the next landmark, this controller progressively align the directions of the mobile base and of the camera. It has to be coupled with the pan-platform controller.

Finallys the robot has to be able to find the next landmark. To do so, the pan-platform is provided a nonzero angular velocity which allows to make it turn until the target is found or until the searching rotation has been completed. During this phase, the mobile base is driven either with an IBVS fed with estimated visual features in the free space or with the path following controller in an obstacle neighborhood.

Now, five separate controllers are available. The global control law will be defined by switching between these controllers depending on the context (see the decision process). To guarantee the global control law continuity at the switching time, we have used an approach, called dynamical sequencing, which allows to insure that the robot velocities provided by both controllers are equal at the switching time (Souères and Cadenat, 2003) (Cadenat et al., 2012).

\subsubsection{Decision}

The decision process defines the navigation strategy which relies on the activation/deactivation of the previously mentioned tools. We have designed a supervision graph for each process. First we focus on the action supervision graphs (see figures 2(a) and 2(b)).

- At the beginning, to compute the path, the robot identifies the landmarks which can be seen from its initial position by performing a $360^{\circ}$ rotation (B0 and C0).

- When it is over (End_rot $=1)$, it waits for the path $T_{P}=\left[T_{P 1}, \ldots, T_{P n_{p}}\right]$ to be computed (B1 and C0).
- When it is available (End_planning =1), the initialization step is realized (B2 and $\mathrm{C} 1$ ) by performing small rotations to estimate the visual features depths of $T_{P 1}$. It ends when the estimation has converged (Depth_conv $=1$ ).

- Now, the first sub-navigation can be launched. The mobile base is controlled using either the IBVS (B3) or the obstacle avoidance (B4) depending on the risk of collision evaluated from laser data (Obs = 1).

- During the sub-navigation, the camera regularly looks for $T_{P 2}(\mathrm{C} 2)$ by scanning the environment. If this latter is detected, a subnavigation with respect to $T_{P 2}$ is executed. If this latter is not found (End_found $=1$ ), the camera remains controlled using either the IBVS (C3) or the obstacle avoidance $(\mathrm{C} 4)$ controllers. Moreover, the depth estimation process is relaunched. When it converges, the robot looks for $T_{P 2}$ one more time. This loop is repeated until: $T_{P 2}$ is found (Land_found $=1$ ) or the sub-navigation is over $\left(\right.$ End_vs $\left._{-} 1\right)$. In this last case, the robot performs a whole turn on itself to try a last time to identify the next target (B0 and $\mathrm{C} 0$ ). If it is not found, the map is updated and a new path is computed. If no path can be computed, we consider that the navigation has failed.

- If $T_{P 2}$ has been found (Land_found $=1$ ), the reorientation step is launched (B5 and C5). The latter is stopped when the robot is oriented towards the landmark $T_{P 2}$ (End_re $=1$ ). Then a subnavigation is launched. This loop is repeated until the robot reaches the final landmark or the navigation fails.

During the navigation, perception modalities may change. Indeed, due to occluding obstacles, the current landmark might not be perceived. Thus the robot uses either the measured visual features or the estimated ones when an occlusion occurs $(\mathrm{Occ}=1)$ (figure 2(c)).

The robot has to localize itself when: (i) the mission begins; and (ii) the camera is looking for the next target (figure 2(d)). In case (i), it has to identify the landmarks which lie in the field of view (Depth_conv =1). The result is used to define the initial nodes of the path. This step is over when the rotation is finished (End_rot $=1$ ). In case (ii), the robot has to determine whether the target in its field of view is the next target or not. It ends when the next landmark is found (Land_found =1) or when the "looking for" step is over (End_look $=1)$.

Finally, we consider the modeling (figure 2(e)) and planning (2(f)) processes. At the end of a subnavigation, two cases may occur. Either the next tar- 
get has been found (Land_found $=1$ ) or not. In the first case, the current target is updated (P1), by considering the next target as the current one. In the second case, a rotation is launched to try to find it again in the robot vicinity. If it fails (Land_found $=0 \&$ End_rot $=1$ ), then the map is updated by suppressing the corresponding connection. Then a new path is planned (P2). If no path can be calculated, we consider the navigation has failed. Otherwise, the new path is performed to reach the desired robot pose.

\section{EXPERIMENTS}

\subsection{The experimental conditions, the environment and its modeling}

The long range navigation experiments were carried out in an indoor environment cluttered by occluding (black) and non occluding (gray) obstacles (figure 3(a)). There are $n_{l}=4$ artificial targets $\left\{T_{1}, T_{2}, T_{3}, T_{4}\right\}$. Prior to the navigation, we have chosen the reference poses $S_{i}^{*}$ (figure 3(a)) and following the reasoning described in part 3.3.2, we have obtained a topological map (figure 3(d)). However, we want to force the robot to update the map and replan the path. To do so, we have deliberately introduced an error in this map by suppressing the link between $N_{1}$ and $N_{3}$. The robot will then be given the graph represented in picture $3(\mathrm{~b})$ instead of the one displayed in figure 3(d). In addition, we have also replaced landmark $T_{2}$ by a target without any pattern. The robot will not then be able to recognize this landmark if it is required for the mission. Finally, the camera has been calibrated considering the lens distortions for an accurate control law computation.

\subsection{Live experiments, results and discussion}

The goal is to reach landmark $T_{4}$, starting from a given initial position. A snapshot of the experimental test is shown in figure 4. A video can be found at the following url: http://youtu. be/OWbEPL $4 \mathrm{x}-\mathrm{Pk}$. As landmark $T_{4}$ cannot be seen from the initial pose (figure 4(a)), a localization step is launched and the robot turns on itself to identify the landmarks lying in its vicinity. After this motion, $T_{1}$ is recognized and a path connecting $T_{1}$ and $T_{4}$ is computed: $\left\{T_{1}, T_{2}, T_{3}, T_{4}\right\} 3$ (b). Then, the mission starts. An IBVS controller is selected to converge towards $T_{1}$ (figure 4(b)). During this motion a pedestrian crosses the robot path, occluding the camera view field (figure 4(c)). The estimated depths and



(a) The experimental environment.

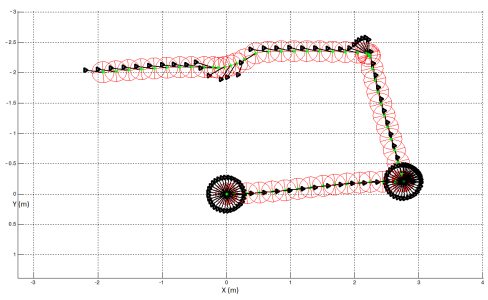

(c) Robot trajectory.

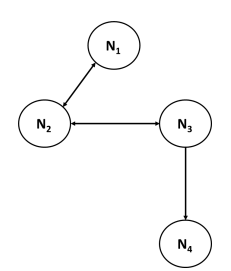

(b) The initial map.



(d) The updated topological map. topological

Figure 3: Experimental data.

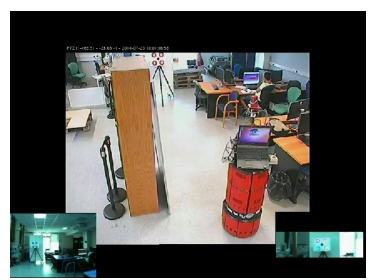

(a)



(c)

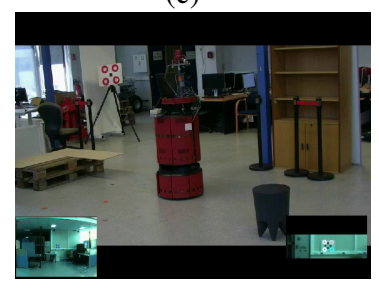

(e)

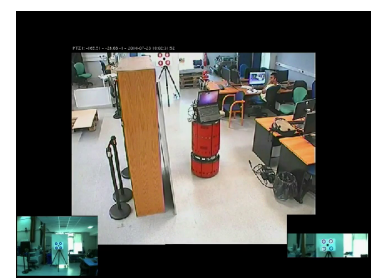

(b)

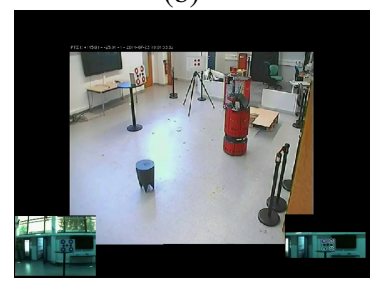

(d)

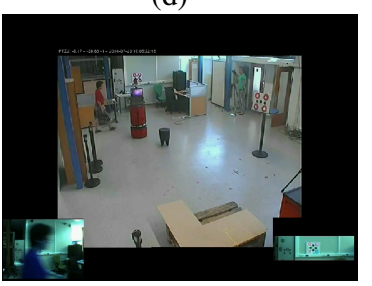

(f)
Figure 4: An experimental test. 
visual features are then used to feed the IBVS controller and the task remains executed, despite the visual features total loss. Once $S_{1}^{*}$ is reached, the robot turns on itself to seek $T_{2}$. It is not found because no pattern has been added to it. Only $T_{3}$ is detected and the map is updated (i.e., the link between $N_{1}$ and $N_{2}$ is deleted while a new one between $N_{1}$ and $N_{3}$ is created). From the current situation, a new path is then planned: $\left\{T_{3}, T_{4}\right\}$ and another IBVS controller is used to converge towards $T_{3}$ (figure $4(\mathrm{~d})$ ). When it is reached, the robot looks for $T_{4}$. When it is found, a final IBVS is launched (figure 4(e)). Two unexpected events then occur (figure 4(f)): first, a non occluding obstacle prevents the vehicle from going towards the goal, but the obstacle avoidance controller is launched, guaranteeing non collision. Second, a pedestrian crosses the robot path, but once again the occlusion problem is successfully treated by our reconstruction algorithms. Finally, despite the presence of humans, initially unmapped obstacles and errors in the map, the mission is completed (Figure 3(c)).

\section{CONCLUSION}

This paper has addressed the navigation problem of a mobile robot in a cluttered environment. First, we have proposed to move from the classical framework to a new one splitting the problem into six processes organized in an architecture. It allows to create new combinations of processes which were not included in the previous framework, guiding more efficiently the elaboration of novel navigation strategies. Second, we have presented our own solution to the navigation problem. It is based on the coupling of sensor-based controllers and of a topological map. We have detailed the processes and the architecture. Finally, we have validated our strategy by showing experimental results. However, to operate in human environments, some improvements can still be made by replacing artificial landmarks by natural ones (SIFT or SURF descriptors), and by considering dynamic environments.

\section{REFERENCES}

Bonin-Font, F., Ortiz, F., and Oliver, G. (2008). Visual navigation for mobile robots : a survey. Journal of intelligent and robotic systems, 53(3):263.

Booij, O., Terwijn, B., Zivkovic, Z., and Krose, B. (2007). Navigation using an appearance based topological map. In IEEE Int. Conf. on Robotics and Automation, pages 3927-3932, Rome, Italy.

Cadenat, V., Folio, D., and Durand Petiteville, A. (2012). A comparison of two sequencing techniques to perform a vision-based navigation task in a cluttered environment. Advanced Robotics.

Chaumette, F. and Hutchinson, S. (2006). Visual servo control, part 1 : Basic approaches. IEEE Robotics and Automation Magazine, 13(4).

Cherubini, A. and Chaumette, F. (2013). Visual navigation of a mobile robot with laser-based collision avoidance. Int. Journal of Robotics Research, 32(2):189-209.

Cherubini, A., Spindler, F., and Chaumette, F. (2011). A redundancy-based approach for visual navigation with collision avoidance. In Computational Intelligence in Vehicles and Transportation Systems (CIVTS), 2011 IEEE Symposium on, pages 8-15. IEEE.

Choset, H., Lynch, K., Hutchinson, S., Kantor, G., Burgard, W., Kavraki, L., and Thrun, S. (2005). Principles of Robot Motion. MIT Press, Boston.

Courbon, J., Mezouar, Y., and Martinet, P. (2009). Autonomous navigation of vehicles from a visual memory using a generic camera model. Intelligent Transport System (ITS), 10:392-402.

Durand Petiteville, A., Durola, S., Cadenat, V., and Courdesses, M. (2013). Management of visual signal loss during image based visual servoing. In Control Conference (ECC), 2013 European, pages 2305-2310. IEEE.

Durand Petiteville, A., Hutchinson, S., Cadenat, V., and Courdesses, M. (2011). 2d visual servoing for a long range navigation in a cluttered environment. In $D e-$ cision and Control and European Control Conference (CDC-ECC), 2011 50th IEEE Conference on, pages 5677-5682. IEEE.

Krajník, T. and Přeučil, L. (2008). A simple visual navigation system with convergence property. In European Robotics Symposium 2008, pages 283-292. Springer.

Matsumoto, Y., Inaba, M., and Inoue, H. (1996). Visual navigation using viewsequenced route representation. In IEEE Int. Conf. on Robotics and Automation, pages 83-88 -2692, Minneapolis, USA.

Royer, E., Lhuillier, M., Dhome, M., and Lavest, J.-M. (2007). Monocular vision for mobile robot localization and autonomous navigation. International Journal of Computer Vision, 74(3):237-260.

Samson, Borgne, and Espiau (1991). Robot control : The task function approach. Oxford science publications.

Segvic, S., Remazeilles, A., Diosi, A., and Chaumette, F. (2009). A mapping and localization framework for scalable appearance-based navigation. Computer Vision and Image Understanding, 113(2):172-187.

Souères, P. and Cadenat, V. (2003). Dynamical sequence of multi-sensor based tasks for mobile robots navigation. In SYROCO, Wroclaw, Poland.

Souères, P., Hamel, T., and Cadenat, V. (1998). A path following controller for wheeled robots wich allows to avoid obstacles during the transition phase. In IEEE, Int. Conf. on Robotics and Automation, Leuven, Belgium. 